\title{
DYSMORPHOLOGY AND TERATOGENESIS
}

\author{
Helen M Kingston
}

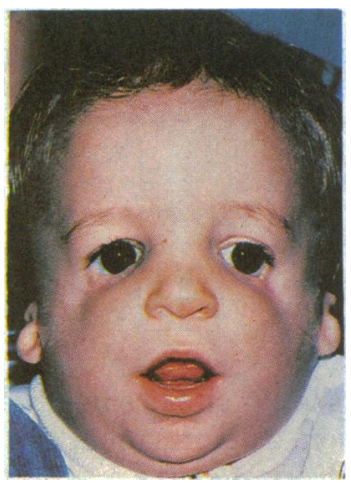

Treacher Collins syndrome: abnormal first branchial arch development giving rise to malar and mandibular hypoplasia with external ear malformations.

Dysmorphology is the study of malformations arising from abnormal embryogenesis. Recognition of patterns of multiple congenital malformations may allow inferences to be made about the timing, mechanism, and aetiology of structural defects. Animal research is providing information about cellular interactions, migration, and differentiation processes and gives insight into the possible mechanisms underlying human malformations. Diagnosing multiple congenital abnormalities in children can be difficult but is important to give correct advice about management, prognosis, and risk of recurrence.

\section{Definition of terms}

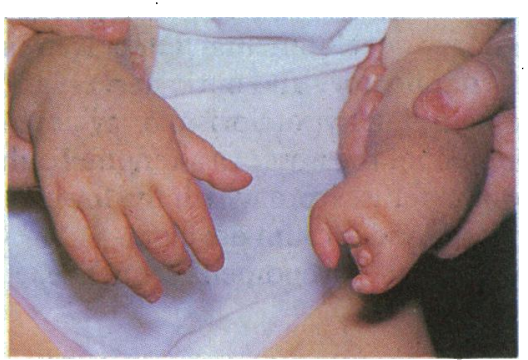

Unilateral terminal transverse defect of the hand occurring as an isolated malformation.

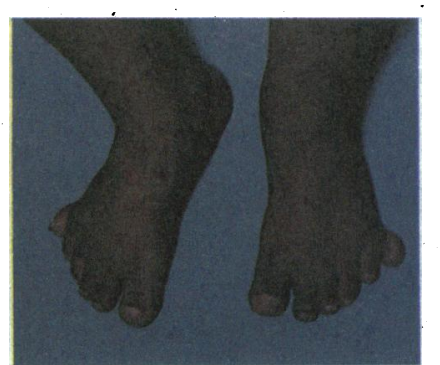

Postaxial polydactyly of the feet in Laurence-Moon-Biedl syndrome lobesity, mental retardation, polydactyly, retinitis pigmentosa, and genita hypoplasia).

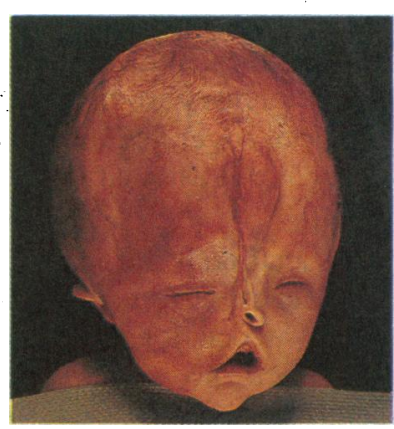

Severe disruption of the face caused by amniotic bands.

\section{Malformation}

A malformation is a primary structural defect occurring during development of an organ or tissue. An isolated malformation, such as cleft lip and palate, congenital heart disease, or pyloric stenosis, can occur in an otherwise normal child. Most single malformations are inherited as polygenic traits with a fairly low risk of recurrence, and corrective surgery is often successful. Multiple malformation syndromes comprise defects in two or more systems and are often associated with mental retardation. The risk of recurrence is determined by the aetiology, which may be chromosomal, teratogenic, due to a single gene, or unknown.

\section{Disruption}

A disruption defect implies that there is destruction of a part of a fetus that had initially developed normally. Amniotic band disruption after early rupture of the amnion is a well recognised entity, causing constriction bands and amputations of digits and limbs and sometimes more extensive disruptions causing, for example, facial clefts and central nervous system defects. As the fetus is genetically normal and the defects are caused by an extrinsic abnormality the risk of recurrence is small. Interruption of the blood supply to a developing part from other causes will also cause disruption owing to infarction with consequent atresia. The prognosis depends solely on the severity of the physical defect.

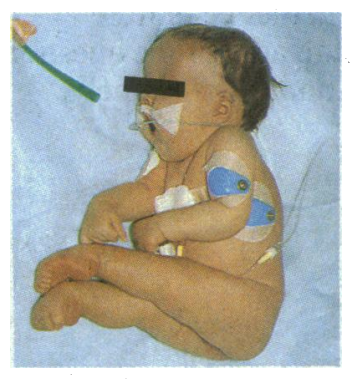

Deformation of legs in newborn infant with hypotonia due to congenital myotonic dystrophy.

\section{Deformation}

Deformations are due to abnormal intrauterine moulding and give rise to deformity of structurally normal parts. Deformations usually involve the musculoskeletal system and may occur in fetuses with congenital neuromuscular problems such as spinal muscular atrophy and congenital myotonic dystrophy. Paralysis in spina bifida gives rise to positional deformities of the legs and feet. In these disorders the prognosis is often poor and the risk of recurrence may be high. 

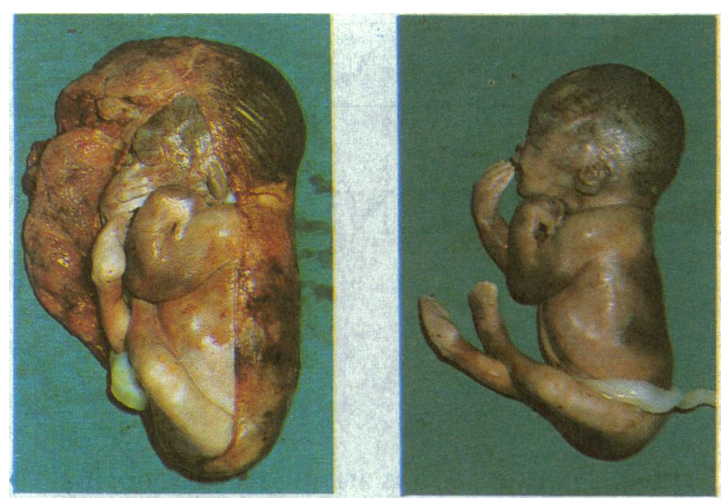

Fetal constraint and deformation due to oligohydramnios in Potter's syndrome (renal agenesis).
Oligohydramnios causes fetal deformation and is well recognised in fetal renal agenesis (Potter's syndrome). The absence of urine production by the fetus results in severe oligohydramnios, which in turn causes fetal deformation and pulmonary hypoplasia. Oligohydramnios caused by chronic leakage of liquor has a similar effect.

A normal fetus may be constrained by uterine abnormalities, breech presentation, or multiple pregnancy. The prognosis is generally excellent, and the risk of recurrence is low except in cases of structural uterine abnormality.

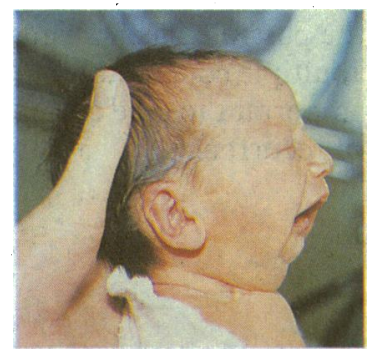

Pierre Robin sequence: mandibular hypoplasia causing cleft palate and respiratory obstruction.

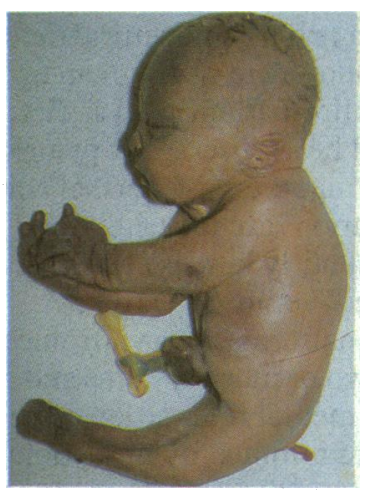

Sirenomelia sequence: fused legs, tail like appendage, absent genitalia, imperforate anus, exomphalos, and renal agenesis.

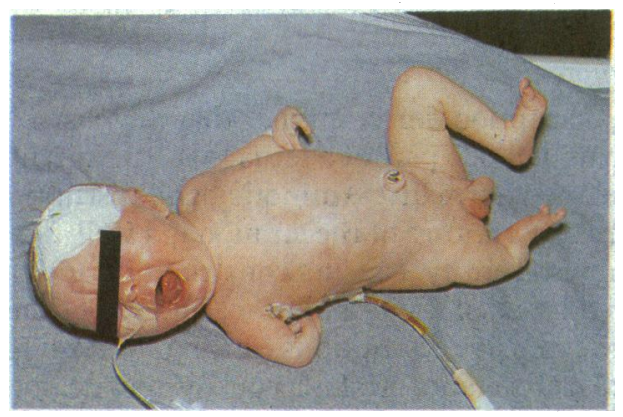

Vater association.

\section{Identification of syndromes}

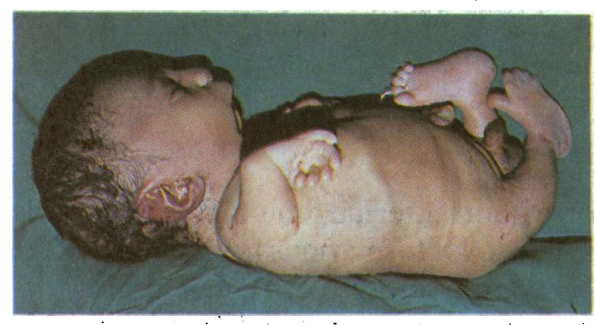

Robert's syndrome: autosomal recessive "pseudothalidomide" syndrome with hypomelia, mid-facial defect, and severe growth deficiency.

\section{Sequence}

The term sequence implies that a series of events occur after a single initiating abnormality, which may be a malformation, a deformation, or a disruption. The features of Potter's syndrome can be classed as a malformation sequence in which the initial abnormality is renal agenesis, which gives rise to secondary deformation and pulmonary hypoplasia. Other examples are the holoprosencephaly sequence and the sirenomelia sequence. In holoprosencephaly the primary developmental defect is in the forebrain, leading to microcephaly, absent olfactory and optic nerves, and midline defects in facial development, including hypotelorism or cyclopia, midline cleft lip, and abnormal development of the nose. In sirenomelia the primary defect affects the caudal axis of the fetus, from which the lower limbs, bladder, genitalia, kidneys, hindgut, and sacrum develop. Abnormalities of all these structures occur in the sirenomelia sequence.

\section{Associations}

Certain malformations occur together more often than expected by chance alone; these are termed associations. The names given to recognised malformation associations are often acronyms of the component abnormalities. Hence the Vater association consists of vertebral anomalies, anal atresia, tracheo-oesophageal fistula, and radial defects. The acronym vacterl has been suggested to encompass the additional cardiac, renal, and limb defects of this association.

Murcs association is the name given to the non-random occurrence of Müllerian duct aplasia, renal aplasia, and cervicothoracic somite dysplasia. In the Charge association the related abnormalities include colobomas of the eye, heart defects, atresia choanae, mental retardation, growth retardation, and ear anomalies.

Patterns of multiple malformations that occur together constitute syndromes. Recognition of syndromes is important to answer the questions that parents of all babies with congenital malformations ask-namely,

What is it?

Why did it happen?

What does it mean for the child's future?

Will it happen again? 

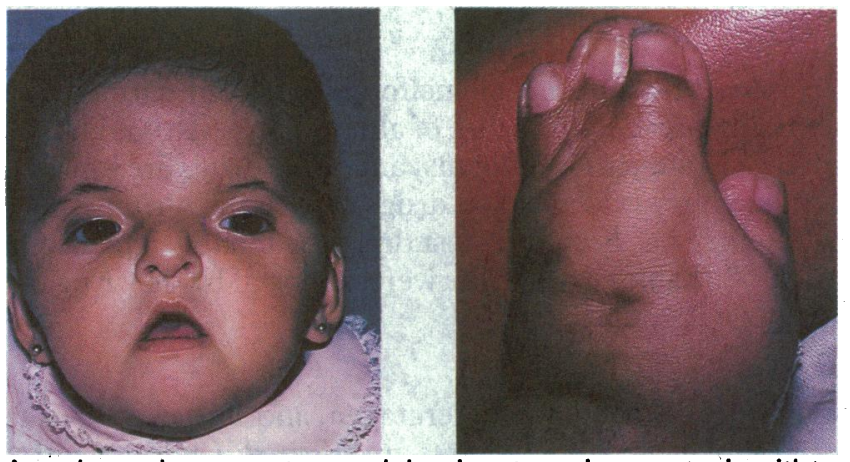

Apert's syndrome: autosomal dominant craniosynostosis with fused digits.
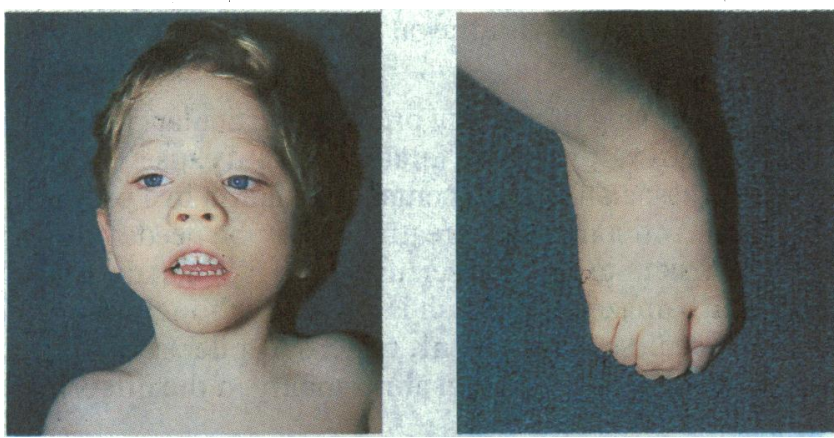

Smith-Lemli-Opitz syndrome: autosomal recessive syndrome with ptosis, anteverted nares, syndactyly of second and third toes, hypospadias, and mental retardation.
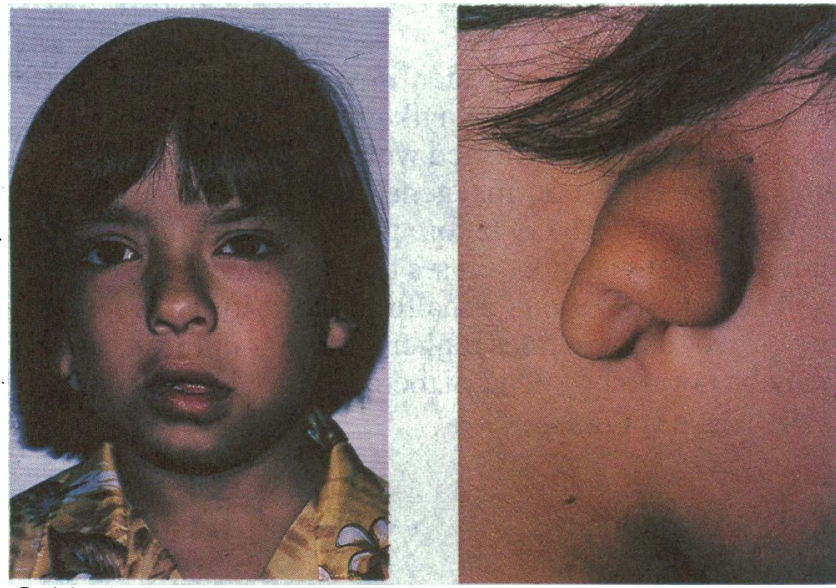

Goldenhar's syndrome (hemifacial microsomia): usually sporadic syndrome with asymmetrical malar, maxillary, and mandibular hypoplasia and microtia.

\section{Stillbirths}

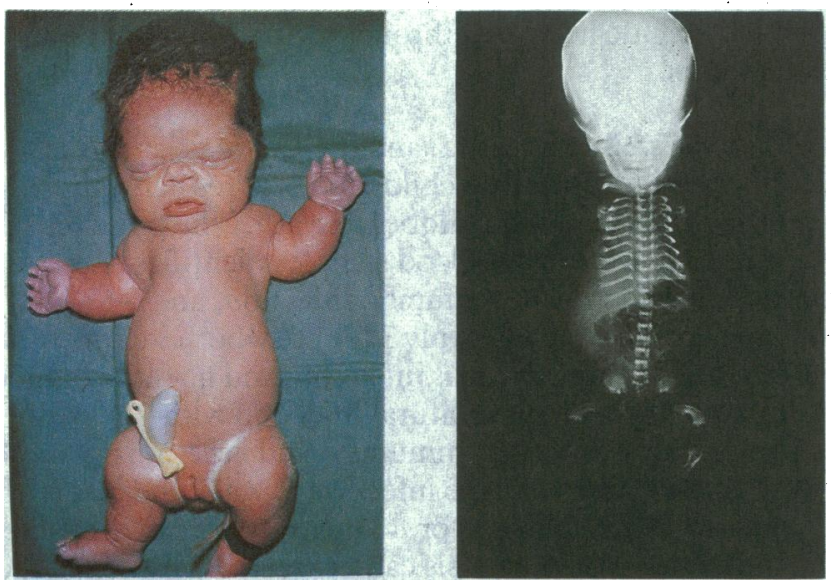

Thanatophoric dwarfism: usually sporadic lethal bone dysplasia.
Parents experience feelings of grief and guilt after the birth of an abnormal child, and time spent discussing what is known about the aetiology of the abnormalities may help to alleviate some of their fears. They also need an explanation of what to expect in terms of treatment, anticipated complications, and long term outlook. Accurate assessment of the risk of recurrence cannot be made without a diagnosis, and the availability of prenatal diagnosis in subsequent pregnancies will depend on whether there is an associated chromosomal abnormality or a structural defect amenable to detection by ultrasonography.

The assessment of infants and children with malformations requires careful taking of a history and a physical examination. Abnormalities during the pregnancy, including possible exposure to teratogens, should be recorded, as. well as the occurrence of any perinatal problems. Parental age and family history may provide clues about the aetiology. Examination of the child should include detailed documentation of the abnormalities present with accurate clinical measurements and photographic records whenever possible, and the investigations required may include chromosomal analysis and biochemical or radiological studies.

A chromosomal or mendelian aetiology has been identified for many multiple congenital malformation syndromes. When the aetiology of a recognised multiple malformation syndrome is not known empirical figures for the risk of recurrence derived from family studies can be used, and these are usually fairly low.

Consanguineous marriages may give rise to autosomal recessive syndromes unique to a particular family: when more than one child is affected counselling the couple using the one in four risk of recurrence associated with autosomal recessive inheritance is appropriate.

Numerous malformation syndromes have been identified, and many are extremely rare.

Published case reports and specialised texts may have to be reviewed before diagnosis. Computer programmes are now available to assist in differential diagnosis, but despite this syndromes in a proportion of children will inevitably remain undiagnosed.

Detailed examination and investigation of malformed stillbirths and fetuses is essential if parents are to be accurately counselled about the cause of the problem, the risk of recurrence, and the availability of prenatal tests in future pregnancies. As with liveborn infants careful documentation of the abnormalities is required with detailed photographic records. Cardiac blood samples and skin biopsy specimens should be taken for chromosome analysis and bacteriological and virological investigations performed. Other investigations, including full skeletal $x$ ray examination and tissue sampling for biochemical studies and DNA extraction, may be necessary. Necropsy will determine the presence of associated internal abnormalities, which may permit diagnosis. 


\section{Environmental teratogens}

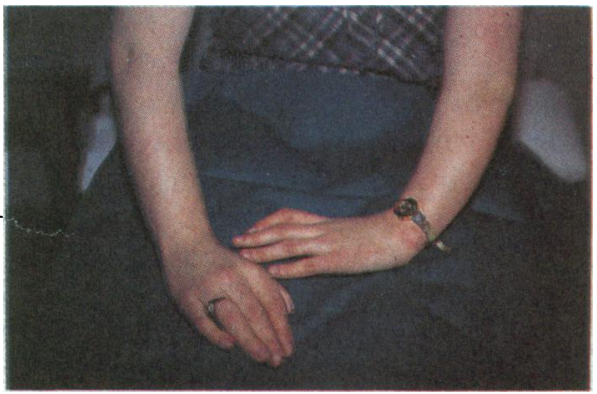

Limb malformation due to intrauterine exposure to thalidomide.

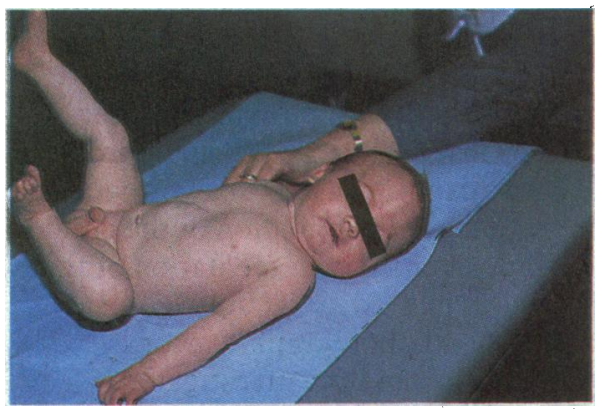

Hypospadias, congenital heart disease, prominent metopic suture (trigonocephaly), and psychomotor retardation in the fetal valproate syndrome.

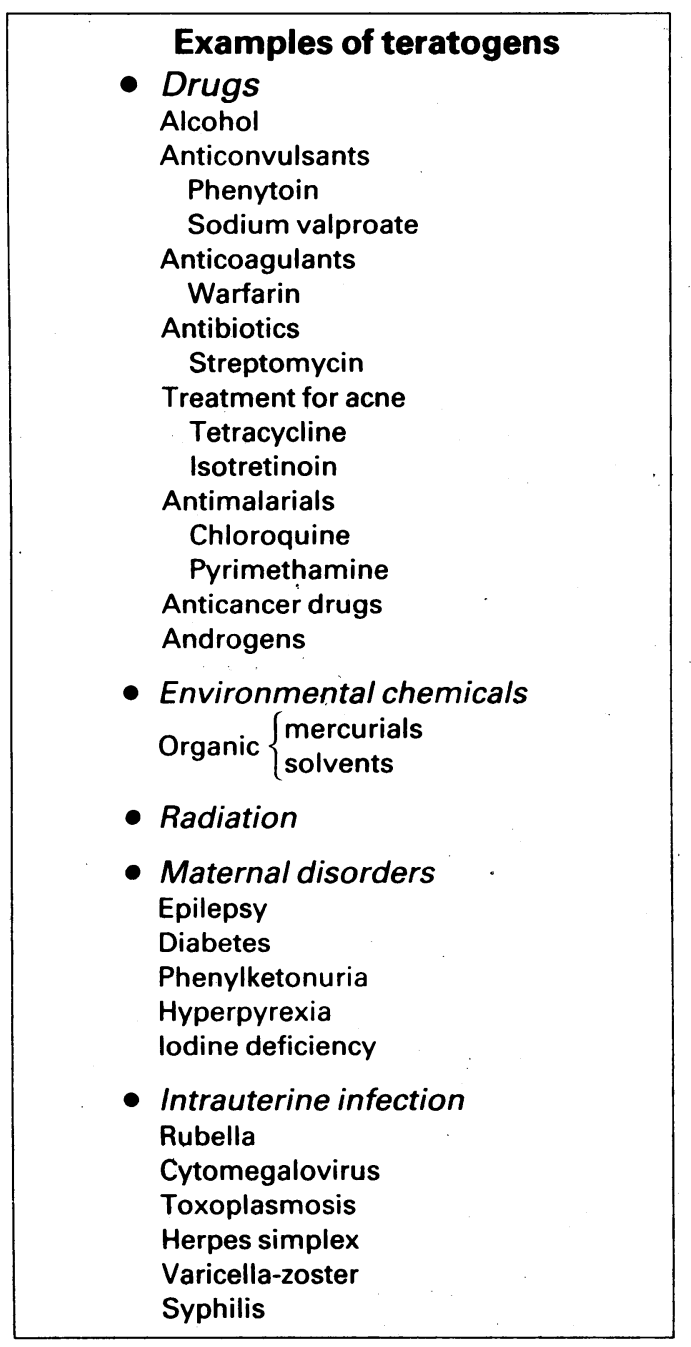

\section{Drugs}

Identification of drugs that cause fetal malformations is important as they constitute a potentially preventable cause of abnormality. Although fairly few drugs are proved teratogens in humans, and some drugs are known to be safe, the accepted policy is to avoid all drugs if possible during pregnancy. Thalidomide has been the most dramatic teratogen identified, and an estimated 10000 babies world wide were damaged by this drug in the early 1960s before its withdrawal.

Alcohol is currently the most common teratogen, and studies suggest that between one in 300 and one in a 1000 infants are affected. Children with the fetal alcohol syndrome exhibit prenatal and postnatal growth deficiency, mental retardation, microcephaly, and characteristic facies with short palpebral fissures, a smooth philtrum, and a thin upper lip. In addition, they have tremulousness owing to withdrawal in the neonatal period.

Treatment of epilepsy during pregnancy presents particular problems as both phenytoin and sodium valproate are teratogenic and cause recognisable syndromes associated with mental retardation in a proportion of exposed pregnancies. Anticonvulsant treatment may be needed during pregnancy to avoid the risk of grand mal seizures, and it is not always possible to change to carbamazepine, which is currently thought to be the most suitable drug. Regardless of treatment, maternal epilepsy has been suggested to increase the risk of congenital abnormality in the offspring.

\section{Maternal disorders}

Several maternal disorders have been identified in which the risk of fetal malformations is increased including phenylketonuria and diabetes: In phenylketonuria the children of an affected woman will be healthy heterozygotes in relation to the abnormal gene, but if the mother is not returned to a carefully monitored diet before pregnancy the high maternal serum concentration of phenylalanine causes microcephaly in the developing fetus. The risk of congenital malformations in the pregnancies of diabetic women is two to three times higher than that in the general population but may be lowered by good diabetic control before conception and during the early part of pregnancy.

\section{Intrauterine infection}

Various intrauterine infections are known to cause congenital malformations in the fetus. Maternal infection early in gestation may cause structural abnormalities of the central nervous system, resulting in neurological abnormalities, visual impairment, and deafness, in addition to other malformations, such as congenital heart disease. When maternal infection occurs in late pregnancy the risk that the infective agent will cross the placenta is higher, and the newborn infant may present with signs of active infection, which include hepatitis, thrombocytopenia, haemolytic anaemia, and preumonitis.

Rubella embryopathy is well recognised, and the aim of vaccination programmes against rubella virus during childhood is at reducing the number of non-immune girls reaching childbearing age. The presence of rubella specific IgM in fetal or neonatal blood samples identifies babies infected in utero. Cytomegalovirus is a common infection, and $5-6 \%$ of pregnant women may become infected. Only 3\% of newborn infants, however, have evidence of cytomegalovirus infection, and no more than $5 \%$ of these develop subsequent problems. Natural infection with cytomegalovirus does not always confer immunity, and occasionally more than one sibling is affected by intrauterine infection. Unlike the case with rubella, vaccines against cytomegalovirus or toxoplasma are not available, and although active maternal toxoplasmosis can be treated with drugs such as pyrimethamine, this carries the risk of teratogenesis. 


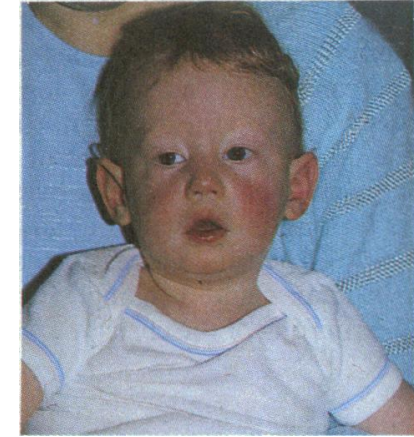

Child with

hepatosplenomegaly, delayed

development, and deafness

due to intrauterine

cytomegalovirus infection.
Herpes simplex infection in the newborn infant is generally acquired at the time of birth, but infection early in pregnancy is probably associated with an increased risk of abortion, late fetal death, prematurity, and structural abnormalities of the central nervous system. Maternal varicella infection may also affect the fetus, causing abnormalities of the central nervous system and cutaneous scars. The risk of a fetus being affected by varicella infection is not known but is probably less than $10 \%$, with a critical period during the third and fourth months of pregnancy. Affected infants seem to have a high perinatal mortality rate.

Dr Helen M Kingston, MD, is consultant clinical geneticist at St Mary's Hospital, Manchester.

The illustrations of disruption of the face caused by amniotic bands, congenital myotonic dystrophy, Pierre Robin sequence, sirenomelia sequence, Smith-Lemli-Opitz syndrome, thalidomide malformation, and the valproate syndrome were reproduced by kind permission of Dr Dian Donnai, St Mary's Hospital, Manchester. The illustrations of Potter's syndrome, the Vater association, and thanatophoric dwarfism were reproduced by kind permission of the University of Manchester and Dr Dian Donnai. The illustrations of Treacher Collins syndrome and Goldenhar's syndrome were reproduced from Dental Update by permission of Update-Siebert Publications.

The $\mathrm{ABC}$ of Child Abuse continues next week. The 11th article in this series will appear on 20 May.

\section{How To Do It}

\section{How to apply for charitable status}

British Association of Cancer United Patients, London EC1M 6AA Maurice Slevin, MD, chairman

Patsy Ryan, RGN

Correspondence to: $\mathrm{Dr}$ Slevin.

BrMed J 1989;298: 1239-40

\author{
Maurice Slevin, Patsy Ryan
}

Creating a unique information service for patients with cancer wasn't Dr Vicky Clement-Jones's only unusual achievement when she founded the British Association of Cancer United Patients (BACUP) in 1984; she also gave a new twist to the old adage "Charity begins at home," as most of the groundwork was done from her home in London while she was convalescing after treatment for advanced ovarian cancer. As her plans gathered momentum and she and willing colleagues were caught up in the frantic merry go round of raising funds, forging contacts, and generally securing a place in the hearts (and pockets) of philanthropic money makers the advantages of acquiring charitable status beckoned invitingly.

Vicky seized all opportunities with characteristic spirit and vigour, and her inspiring enthusiasm (coupled with a sense of true urgency as her prognosis was uncertain) led to the association being registered as a charity in just three days. The year of 1984 certainly had its fair share of unique achievements.

\section{Pros and cons}

The first question a potential charity crusader should ask, uncharitably enough, is, "How will charitable status give my plans the edge?" The solution comes from objectively weighing up the pros and cons and banishing Dickensian overtones of the term charity.

The major advantage is financial. The great British public is reassured by the respectability of a registered charity number when being persuaded to part with hard earned cash. Even if you don't envisage rattling collecting tins at Saturday afternoon shoppers, when you are appealing to benevolent businesses and charitable foundations the magic number proves an effective open sesame to their coffers. More pragmatically, registered charities are automatically entitled to $50 \%$ rates relief on premises-some generous local authorities even offer $100 \%$ relief. Some useful exemptions from value added tax and stamp duty relax tense purse strings, and opportunities for donations free of tax gild the carrot to tempt valuable regular donors.

The commonest drawback to attaining charitable status is the strict legal limitations then imposed on political and campaigning activities. This is sensitive territory, and if planning to venture into it you would be well advised to have the guiding hand of a good lawyer at your elbow.

Your best guide is generally a solicitor experienced in all aspects of forming charities. The increasingly complex charity laws are a specialist subject, and experts tend to be thin on the ground. Your willing regular solicitor, however, could do the job ably assisted by the Charity Law Advisory Service for Solicitors. This is a service to which lawyers can turn for practical and expert help with charity law. The National Council for Voluntary Organisations can also give legal advice and supply model constitutions for budding founders of a charity. 